\title{
The proportion of ocular surface diseases in untreated patients with epiphora
}

This article was published in the following Dove Press journal:

Clinical Ophthalmology

\author{
Sho Ishikawa' \\ Kouichiro Murayama² \\ Naoko Kato' \\ 'Department of Ophthalmology, \\ Saitama Medical University, Saitama, \\ Japan; ${ }^{2}$ Tsuruse Murayama Eye Clinic, \\ Saitama, Japan
}

\begin{abstract}
Purpose: Various ocular surface disorders, such as dry eye, infectious and non-infectious inflammation, anatomical anomalies in lid structure, and/or obstruction of the tear outflow tract, can cause epiphora. However, few reports have described the proportions of causative diseases in patients with epiphora. Therefore, we investigated the frequent causes of epiphora in older adult patients.
\end{abstract}

Materials and methods: We enrolled 220 patients ( 70 men, 150 women) who visited a primary eye care clinic from January 2016 to August 2017 with lacrimation as a chief complaint. The mean age of the patients was $61.3 \pm 10.9$ years. The causes of epiphora were assessed by slit-lamp examinations, dry eye tests, fluorescein disappearance tests, and lacrimal pathway washing.

Results: The causes of epiphora were dry eye in 96 , conjunctivochalasis in 56, lacrimal obstruction in 30 , conjunctivitis in 20 , trichiasis in 16 , and facial palsy in 2 patients. Ocular surface diseases were identified in 187 of the $220(85 \%)$ patients who complained of epiphora. Conjunctivochalasis was observed in 36 of the 96 (38\%) patients with dry eye. Among the patients with dry eye, $99 \%$ had short break-up time type dry eye, with the random break type being the most frequent type $(61 / 96 ; 64 \%)$ in patients with epiphora.

Conclusion: Among older adult patients whose main complaint was epiphora, $85 \%$ had ocular surface diseases. In contrast, only $15 \%$ of patients had lacrimal diseases. Short break-up time dry eye, especially the random break type, was the most frequently observed form of dry eye in our cohort.

Keywords: epiphora, dry eye, ocular surface disorder, break-up time, lacrimal obstructive disease

\section{Introduction}

Epiphora, or lacrimation, is one of the most common complaints of patients who visit primary eye care clinics. Causes of epiphora include ocular surface disorders, such as dry eye, infectious and non-infectious inflammation, anatomical anomalies in the lid structure, and/or obstruction of the tear outflow tract. Reflex hypersecretion and/or disorders of the lacrimal pathway are also associated with epiphora. For example, it was reported that about $50 \%$ of patients with dry eye complain of epiphora. ${ }^{1}$ Although epiphora is not a vision-threatening disorder, it can have a negative effect on patients' quality of life. ${ }^{2}$ In particular, epiphora causes discomfort and interferes with daily activities, such as reading, driving, working on a computer, and watching television. ${ }^{2}$ Therefore, ensuring that epiphora is effectively managed is important for improving patients' quality of life, especially in middle-aged or older adults. However, few investigations in the epidemiological literature have examined the causes of epiphora owing to its complexity. Indeed, it is difficult to distinguish the causes of epiphora, as there may be several underlying causes, the patient may have a history of eye drop 
treatment for other diseases, and the examinations for epiphora can only be performed by a specialist.

In the present study, we retrospectively investigated the proportions of various etiological diseases in older adult patients who visited a primary eye care clinic owing to epiphora.

\section{Materials and methods Patients}

The study was approved by the ethical committee of the Saitama Medical University (17-140) and was conducted in accordance with Declaration of Helsinki provisions. Patient informed consent was waived by the ethical committee of the Saitama Medical University because of the retrospective design of the study. A retrospective analysis was conducted by collecting data from the medical records of patients who visited a private eye clinic, namely, Tsuruse Murayama Eye Clinic, from January 2016 to August 2017, with epiphora as their chief complaint. The collected data were checked, analyzed, and confidentiality was maintained, and the data files were kept securely. Patients $>20$ years of age were included. Patients using an ocular medication or with a history of ocular surface surgery were excluded. In total, 220 patients (70 men, 150 women; mean age $61.3 \pm 10.9$ years) were evaluated.

All patients underwent complete ophthalmologic examinations, including slit-lamp examinations, dry eye tests, fluorescein disappearance tests (FDTs), and lacrimal pathway washing.

\section{Slit-lamp examination}

Patients' lid position anomalies, eyelash anomalies, blepharitis, allergic conjunctivitis, infectious conjunctivitis, conjunctivochalasis, facial palsy, and punctal stenosis or obstruction were evaluated. Conjunctivochalasis grading was also performed, as follows: grade 0 , no fold; grade 1 , a single, small fold; grade $2,>2$ folds, not higher than the tear meniscus; and grade 3, multiple folds, higher than the tear meniscus. ${ }^{3}$ Only eyes with grade 3 were regarded as having conjunctivochalasis.

\section{Dry eye test}

Dry eye was diagnosed according to the Dry Eye Workshop II diagnostic criteria, ${ }^{4}$ which included: 1$)$ the presence of dry eye symptomatology, 2) qualitative or quantitative disturbance of the tear film (break-up time [BUT] $<10$ seconds) and 3) the presence of ocular surface staining. A patient was diagnosed with definite dry eye only if they fulfilled criterion 1 along with one of 2 or 3 criteria. ${ }^{4}$ We used Ocular Surface Disease
Index (OSDI) ${ }^{5}$ for screening dry eye. We considered OSDI score $\geq 13$ as abnormal. Patient fluorescein staining scores were evaluated and the BUTs were measured. Fluorescein staining scores were rated on a scale of $0-9$, with scores $\geq 3$ being considered abnormal. ${ }^{4}$ Fluorescein tear film BUT was measured with a fluorescein solution without anesthesia. For this test, subjects were instructed to blink several times to ensure adequate mixing of the $1 \%$ fluorescein dye into the tear film. The interval between the last complete blink and the appearance of the first corneal black spot in the stained tear film was measured 3 times, and the average value was utilized in the statistical analysis. A fluorescein tear film BUT $<10$ seconds was considered abnormal. ${ }^{4}$ We especially diagnosed short-BUT-type dry eye with fluorescein tear film BUT $<5$ seconds. We classified the BUT patterns into 5 break types, namely the area, spot, line, dimple, and random break types. ${ }^{6}$

\section{Fluorescein disappearance test}

In the FDT, $2 \mu \mathrm{L}$ of eye drops containing $1 \%$ fluorescein solution was administered to each patient, and after 5 minutes, the color of the fluorescein remaining in the tear film was assessed. The FDT was considered positive when the fluorescence color did not change. ${ }^{7}$

\section{Lacrimal pathway washing}

When the FDT was positive, the lacrimal pathway was washed to determine whether the lacrimal pathway was obstructed. To do this, a 23G Nakamura's lacrimal washing single-size needle ${ }^{\circledR}$ (Inami, Tokyo, Japan) filled with saline was used. A patient was diagnosed with lacrimal obstruction when the saline did not reach their nasal cavity.

\section{Statistical analysis}

All statistical analyses were performed using JMP version 10 software $^{\circledR}$ (SAS Institute, Tokyo, Japan). The associations between the FDT results and lacrimal obstructive diseases were examined using the chi-squared test. Statistical significance was set at $P<0.05$. Note that $P$-values smaller than 0.001 are listed as $P<0.001$.

\section{Results}

The cause of epiphora was determined to be dry eye in 96 (44\%), conjunctivochalasis in $56(25 \%)$, lacrimal obstruction in $30(14 \%)$, conjunctivitis in $20(9 \%)$, trichiasis in $16(7 \%)$, and facial palsy in 2 patients (1\%) (Table 1$)$.

Of the 220 patients, 96 (44\%) were diagnosed with dry eye, and 95 of these 96 patients (99\%) had short BUT type 
Table I Causes of epiphora

\begin{tabular}{llll}
\hline & Patients (\%) & Male & Female \\
\hline Dry eye & $96(44 \%)$ & 18 & 78 \\
Conjunctivochalasis & $56(25 \%)$ & 24 & 32 \\
Lacrimal obstruction & $30(14 \%)$ & 7 & 23 \\
Conjunctivitis & $20(9 \%)$ & 9 & 11 \\
Trichiasis & $16(7 \%)$ & 10 & 6 \\
Facial Palsy & $2(1 \%)$ & 2 & 0 \\
\hline
\end{tabular}

dry eye. Regarding the fluorescein break-up pattern, the random break type was the most frequently observed pattern (64\%) in patients with epiphora (Table 2).

Of the 20 patients with conjunctivitis, 12 had allergic conjunctivitis and 8 had epidermic keratoconjunctivitis. None of the patients exhibited eyelid anomalies. Nasolacrimal duct obstruction was identified in $22(10 \%)$, common canalicular obstruction was found in $6(3 \%)$, and punctal obstruction was observed in 2 patients (1\%).

All patients underwent the FDT (Table 3). The FDT was positive in 48 of the 220 patients (22\%). Among the patients with positive FDT results, 30 (63\%) had lacrimal obstructive diseases and 18 (37\%) did not (14 patients [29\%] had conjunctivochalasis, 2 [4\%] had exotropia, and 2 [4\%] had facial palsy). In contrast, none of the patients with negative FDT results had lacrimal obstructive diseases. A positive FDT was significantly associated with the presence of lacrimal obstructive diseases $(P<0.001)$. The sensitivity of FDT for lacrimal obstructive diseases was $100 \%$ and the specificity was $91 \%$.

\section{Discussion}

In the present study, we evaluated the etiology of untreated epiphora in older adults. Our data revealed that about $85 \%$ of the patients had ocular surface diseases and 15\% had lacrimal diseases. Moreover, the FDT was positive in $22 \%$ of patients. The sensitivity of FDT for lacrimal obstruction was $100 \%$ and specificity $91 \%$. To our surprise, a lot of patients who did not receive treatment for epiphora had ocular surface diseases. FDT was thought to be useful to distinguish the patients, who had lacrimal obstructive diseases, from those who had not.

Table 2 Type of BUT

\begin{tabular}{llllll}
\hline BUT type & Random & Spot & Dimple & Line & Area \\
\hline Number & 61 & 18 & 8 & 8 & 1 \\
BUT (sec) & $4.1 \pm 0.8$ & 0 & $1.8 \pm 0.9$ & $1.0 \pm 0.8$ & 0 \\
Fluorescein stain score & $0.5 \pm 0.5$ & $0.5 \pm 0.5$ & $0.5 \pm 0.4$ & $0.7 \pm 0.5$ & 5
\end{tabular}

Abbreviation: BUT, break-up time.
Table 3 Correlation between FDT and lacrimal obstructive diseases

\begin{tabular}{llll}
\hline & \multicolumn{3}{l}{ Lacrimal obstructive diseases } \\
\cline { 2 - 4 } & + & - & Total \\
\hline FDT+ & 30 & 18 & 48 \\
FDT- & 0 & 172 & 172 \\
& 30 & 190 & 220 \\
\hline
\end{tabular}

Abbreviation: FDT, fluorescein disappearance test.

Previously, Mainville and Jordan ${ }^{8}$ reported that lacrimal obstruction and dry eye-related reflex tear secretion occurred in $48.7 \%$ and $40 \%$ of patients with epiphora, respectively. Similarly, Ulusoy et $\mathrm{al}^{9}$ reported that lacrimal obstruction and dry eye-related reflex tear secretion occurred in $48.4 \%$ and $38.7 \%$, of patients with epiphora, respectively. Williams et $\mathrm{al}^{10}$ reported slightly lower prevalence of $33.3 \%$ and $22.0 \%$ for lacrimal obstruction and dry eye-related reflex tear secretion, respectively, in patients with epiphora. Unlike these previous studies, the most frequently observed cause of epiphora in the present study was dry eye-related reflex tear secretion $(85 \%)$, while lacrimal obstruction was observed in only $15 \%$ of the enrolled patients. The discrepancies between our findings and those reported previously may be related to the study setting and type of patient enrolled; while the previous investigations were performed in tertiary oculoplastic practices or ophthalmology clinics and included patients who had been treated previously, the present study was performed in a private eye clinic and included only untreated patients. Our study setting and patient inclusion criteria likely led us to enroll patients with a greater variety of diseases in the present study.

Here, the most frequency observed disorder among patients whose chief complaint was epiphora was short BUT type dry eye (44\%), especially the random break type. According to a clinical survey in Japan, the most prevalent subtype of dry eye is aqueous-deficient dry eye $(35.0 \%)$, followed by short BUT type dry eye (26.7\%). ${ }^{11}$ In contrast, short BUT type dry eye patients were 95 (99\% of dry eye patients) and aqueous-deficient dry eye was only $1(1 \%)$ in this study. Moreover, short BUT type dry eye with minimally decreased tear production and minimal vital staining may be associated with severe symptoms. ${ }^{12}$ In short BUT type dry eye, the random break type is caused by the increased evaporation of aqueous tears and dysfunction of the lipid layer. ${ }^{6,13}$ In a prior report, the lipid layer thickness, as evaluated using DR-1a (KOWA, AIchi, Japan), was negatively correlated with Schirmer's test value,${ }^{14}$ while another study found that tear fluid production was increased as a compensatory response 
to meibomian gland loss and high evaporative type of dry eye. ${ }^{15}$ These reports indicate that the lack of the lipid layer and evaporative type of dry eye may enhance reflex tearing. Based on our data, we believe these features of short BUT type dry eye and increased evaporative-type dry eye caused epiphora.

In this study, conjunctivochalasis was found in 56 patients and trichiasis in 16; however, none of the patients had entropion or ectropion. It was previously reported that eyelid disorders, including entropion, ectropion, trichiasis, and conjunctivochalasis, may induce epiphora. ${ }^{16}$ Ectropion may cause the punctum to move away from the bulbus, thus obstructing lacrimal drainage. In entropion, the lid margin turns inward toward the ocular surface and may damage the cornea and conjunctiva, causing reflex tearing. Additionally, conjunctivochalasis may cause epiphora by mechanically displacing the normal tear meniscus and impeding tear flow along the eyelid margin toward the punctum. ${ }^{16}$

In our study, we found that the FDT was a useful examination for identifying lacrimal obstructive diseases. It is known that FDT is a useful examination for identifying lacrimal obstructive diseases. According to Zappia and Milder, ${ }^{7}$ the positive rate of the FDT for lacrimal diseases is 95\%. In the present study, the FDT was positive in all patients with lacrimal disease. Fourteen patients with conjunctivochalasis, 2 with exotropia, and 2 with facial palsy also had a positive FDT. In these disorders, tear clearance and rapid tear flow after blinking (tear Krehbiel flow) ${ }^{17}$ may be compromised. In patients with conjunctivochalasis or facial palsy, blinking is sometimes incomplete and the rapid tear flow after blinking may not be induced. As the tear Krehbiel flow is faster in medial gaze positions than it is in the frontal gaze position, ${ }^{18}$ tear clearance is also lower than it is when the eyes are in a normal position in patients with exotropia. These dysfunctions may cause some patients without lacrimal obstruction to show false positive FDT.

The present study possessed several limitations. First, there was no patient with punctum stenosis in this study. We considered punctum stenosis as $23 \mathrm{G}$ lacrimal washing single-size needle not being able to pierce a patient's punctum. Although we washed lacrimal pathway of all patients with the $23 \mathrm{G}$ lacrimal washing single-size needle, there was no patient with punctum stenosis. Second, we did not use special examinations for diagnosis, classification, and severity of dry eye in the present investigation, such as measurement of tear osmolarity, tear meniscus height, and meibomian gland dysfunction grade and severity. However, according to Yokoi et al, the random break pattern is a result of the evaporation of aqueous tear, ${ }^{6}$ so we considered those eyes may possess the evaporative-type dry eye in the present study.

\section{Conclusion}

We evaluated the etiology of untreated patients with epiphora who visited a private eye clinic and found that $\sim 85 \%$ of patients had ocular surface diseases, especially short BUT type dry eye. Disorders of the lacrimal drainage system were only observed in $15 \%$ of the patients. Furthermore, our findings support that FDT is an easy but valuable test for differentiating lacrimal obstructive disease as the main cause of epiphora from other ocular surface disorders.

\section{Author contributions}

All authors contributed toward data analysis, drafting, and critically revising the paper, and agreed to be accountable for all aspects of work.

\section{Disclosure}

The authors report no conflicts of interest in this work.

\section{References}

1. Tsubota K, Yokoi N, Shimazaki J, et al. New Perspectives on Dry Eye Definition and Diagnosis: A Consensus Report by the Asia Dry Eye Society. Ocul Surf. 2017;15(1):65-76.

2. Shin JH, Kim YD, Kl W; Korean Society of Ophthalmic Plastic and Reconstructive Surgery (KSOPRS). Impact of epiphora on visionrelated quality of life. BMC Ophthalmol. 2015;15:6.

3. Meller D, Tseng SC. Conjunctivochalasis: literature review and possible pathophysiology. Surv Ophthalmol. 1998;43(3):225-232.

4. Wolffsohn JS, Arita R, Chalmers R, et al. TFOS DEWS II Diagnostic Methodology report. Ocul Surf. 2017;15(3):539-574.

5. Schiffman RM, Christianson MD, Jacobsen G, Hirsch JD, Reis BL. Reliability and validity of the Ocular Surface Disease Index. Arch Ophthalmol. 2000;118(5):615-621.

6. Yokoi N, Georgiev GA, Kato H, et al. Classification of Fluorescein Breakup Patterns: A Novel Method of Differential Diagnosis for Dry Eye. Am J Ophthalmol. 2017;180:72-85.

7. Zappia RJ, Milder B. Lacrimal drainage function. 2. The fluorescein dye disappearance test. Am J Ophthalmol. 1972;74:160-162.

8. Mainville N, Jordan DR. Etiology of tearing: a retrospective analysis of referrals to a tertiary care oculoplastics practice. Ophthalmic Plast Reconstr Surg. 2011;27(3):155-157.

9. Ulusoy MO, Kıvanç SA, Atakan M, Akova-Budak B. How Important Is the Etiology in the Treatment of Epiphora? J Ophthalmol. 2016;2016: 1438376.

10. Williams B, Johnson D, Hurst J, Kratky V. Patterns and causes of epiphora referrals to a tertiary oculoplastic practice. Can J Ophthalmol. 2014;49(2):180-182.

11. Kawashima M, Yamada M, Suwaki K, et al. A Clinic-based Survey of Clinical Characteristics and Practice Pattern of Dry Eye in Japan. Adv Ther. 2017;34(3):732-743.

12. Toda I, Shimazaki J, Tsubota K. Dry eye with only decreased tear break-up time is sometimes associated with allergic conjunctivitis. Ophthalmology. 1995;102(2):302-309.

13. Yokoi N, Uchino M, Uchino Y, et al. Importance of tear film instability in dry eye disease in office workers using visual display terminals: the Osaka study. Am J Ophthalmol. 2015;159(4):748-754. 
14. Arita R, Morishige N, Fujii T, et al. Tear Interferometric Patterns Reflect Clinical Tear Dynamics in Dry Eye Patients. Invest Ophthalmol Vis Sci. 2016;57(8):3928-3934.

15. Arita R, Morishige N, Koh S, et al. Increased Tear Fluid Production as a Compensatory Response to Meibomian Gland Loss: A Multicenter Cross-sectional Study. Ophthalmology. 2015;122(5):925-933.

16. Tse DT, Erickson BP, Tse BC. The BLICK mnemonic for clinicalanatomical assessment of patients with epiphora. Ophthalmic Plast Reconstr Surg. 2014;30(6):450-458.
17. Yamaguchi M, Ohta K, Shiraishi A, et al. New method for viewing Krehbiel flow by polymethylmethacrylate particles suspended in fluorescein solution. Acta Ophthalmol. 2014;92(8):e676-e680.

18. Zheng X, Yamaguchi M, Kamao T, et al. Visualization of Tear Clearance Using Anterior Segment Optical Coherence Tomography and Polymethylmethacrylate Particles. Cornea. 2016;35(Suppl 1):S78-S82.

\section{Publish your work in this journal}

Clinical Ophthalmology is an international, peer-reviewed journal covering all subspecialties within ophthalmology. Key topics include: Optometry; Visual science; Pharmacology and drug therapy in eye diseases; Basic Sciences; Primary and Secondary eye care; Patien Safety and Quality of Care Improvements. This journal is indexed on

Submit your manuscript here: http://www.dovepress.com/clinical-ophthalmology-journal

\section{Dovepress}

PubMed Central and CAS, and is the official journal of The Society of Clinical Ophthalmology (SCO). The manuscript management system is completely online and includes a very quick and fair peer-review system, which is all easy to use. Visit http://www.dovepress.com/ testimonials.php to read real quotes from published authors. 